\title{
Recent Advances of Sensors for Assistive Technologies
}

\author{
Wei (Vivien) Shi \\ Department of Engineering \& Technology, University of Wisconsin-Stout, Menomonie, WI 54751, USA \\ Email: shiw@uwstout.edu
}

Received March 2015

\begin{abstract}
In this paper, the recent advances of sensors incorporated in assistive technologies are presented. Based on the function and operation of modern assistive devices, a variety of sensors are described with their features and applications. Further improvements and future trend are pointed out and discussed.
\end{abstract}

Keywords

Assistive Robot, Assistive Technology, Sensor

\section{Introduction}

The world's population age 65 and older is growing by an unprecedented rate. An aging population with a longer life expectancy results in a larger population of fragile elderly, the chronically ill and those requiring rehabilitation [1]. In 2012, the percentage of the world population who are more than 65 was $6.9 \%$, and this is estimated to increase to around $20 \%$ by 2050 [2]. Due to the increase in this proportion of the population, this has created a growing need for innovative approaches to deliver care services for older adults. In the next two decades there will be a significant increase in the elderly population and this in turn will result in a much greater need for effective assistive devices.

In addition, persons with disabilities are less likely to be employed. A 2013 study showed that $46 \%$ of the people ages 21 - 64 with a disability were employed and a significant number of those were employed part time. These figures compare with an employment rate of $84 \%$ for those without a disability [3]. These population demographics clearly indicate that over the next 20 years there will be a rapidly expanding need for assistive devices of all types.

Various governments around the world are investigating various methods of keeping the elderly and disabled people involved socially and economically for longer in addition to reducing the number of them needing institutionalization. With improved sensing and communication technologies, motion tracking has made assistive living and environment possible [4]. Assistive environment provides many health care solutions including in-home care for the elderly and the disabled.

Sensors have four main components: sensing, processing, communication, and energy/power units. Body sensors fall into two main categories, implantable and wearable. The former measure parameters inside the body and mostly operate as interfaces to relatively small software components attached to or implanted into human 
bodies. The implantable sensors provide bidirectional communication interfaces between a person and a remote information system that provides healthcare services, diagnosis, or upgrades [2]. Wearable sensors, although not as invasive as their implantable counterparts, nevertheless must withstand the human body's normal movements and infringe on them as little as possible [5].

Efficient sensors with the assistive devices could enable early disease detection, remote diagnosis and the independent living of elderly people and chronically ill patients [6]. Recent developments in ambient assistive living technologies have demonstrated the feasibility of using ambient sensors in supporting independent living [7]. Unlike wearable sensors, they tend to have more battery and processing capacities, but have limited use in capturing physiological information or in multiple occupancy dwellings.

The purpose of this work is to present recent advances, development and application of sensors for assistive devices that have been used for aged care and assisting persons with disabilities. Therefore, researchers and practitioners will be aware of the challenges in this area. The paper is organized as: Section 2 presents a variety of sensors applied in assistive technologies; finally, Section 3 concludes the paper.

\section{Sensors Used for Assistive Technologies}

The definition "assistive technologies as a product, equipment or device, usually electronic or mechanical in nature, which helps people with disabilities to maintain their independence or improve their quality of life", has been given by the Australian Dementia Resources Guide in 2008 [8]. This definition has extended the use of assistive technologies from devices to help adults with disabilities to products facilitating the seniors' daily lives [9]. As the sensor technology advances, assistive devices have been able to detect various kinds of physiological variables.

Wearable devices may be categorized according to their functional aim, including monitoring system, iLife fall detection sensors [10] [11] recognize and react to falls, Health buddy which measures and records vital signs, PROACT [12] [13] glove which monitors contact with everyday objects and SenseCam [14] [15] which improves retrospective memory.

\subsection{Sensors Incorporated in Assistive Robots}

Robotic technology for supporting human activities, such as intelligent wheel chairs [16] [17], prosthetic limbs [18] [19], and wearable robots [20], have been studied for their practical use in various aspects of daily life. As mobility declines it becomes increasingly difficult to maintain independence. Increasingly assistive devices for mobility are proposed, examples include the robot suit, "designed to help the elderly and enfeebled to walk and carry heavy objects", which is designed to support bodyweight, reduce stress on the knees, help people climb steps and stay in crouching positions.

In [21], three infrared (IR) sensors are mounted on the robot hand as Figure 1, in order to control the robot

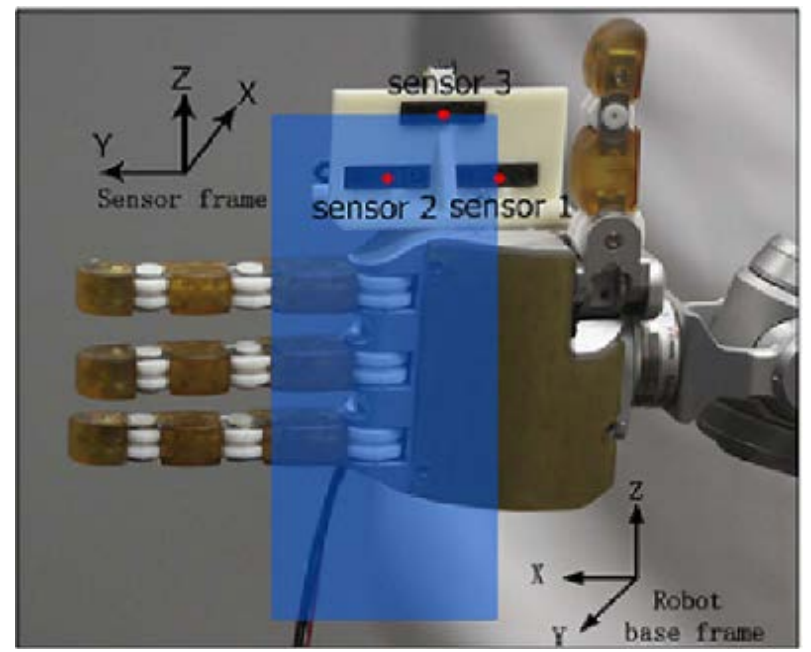

Figure 1. Hardware of IR sensors [21]. 
hand to grasp objects using the information from the sensors readings and the interface component. The IR sensors are used for proximally sensing the object distance and providing a corrective signal for the hand to close in on the object. Besides, tactile sensors are employed in the system with contact based sensing methods.

The first capability is that the hand can autonomously move to a suitable pre-grasp position if at least one of the three sensors detects the object. In the pre-grasp position, the robot can directly close the hand to grasp the object. The second functionality of the system is collision avoidance, for the reason that it will keep a distance to any object. With more sensors detecting the object, the distance would be larger. Thirdly, the system is robust. The end-effector even can track a mobile object. KINECT is employed as the sensor to collect human joint position data, then the robot could mimic human after mapping between their joint coordinates. At the same time, the human hand gesture could be recognized as open or closed, which could provide the signal for the robot to open or close hand.

Any sensor is designed to detect and locate objects and to provide user with information that allows him to determine the dimension and height of the object, its position and direction of movement. Pre-touch sensing, whose ranges are between tactile sensors and vision, has been employed for short range perception. [22] [23] describe Electric Field pre-touch which aligns robot arms with objects and fits the fingers for pre-shaping.

Optical infrared sensors are introduced for pre-touch during final grasp adjustments. The method in [24] detects the orientation of an object surface using the IR sensors that fit inside the fingers. However, [24] can only adjust the fingers, and [25] can only be used for one dimension of the end-effector. [26] equips IR sensors on a gripper to fit the griper for a normal force to the object boundary.

In [27], autonomic sensing is proposed for sensor networks. Autonomic sensing enables the assistive robots to be self-managed, self-configured, self-optimised, and self-adapted. Allowing managing itself will enable unobtrusive sensing and facilitate the adoption of the technology for long-term care applications. A novel self-organizing sensor network is designed for a home monitoring application with both wearable and ambient sensors based on cluster network architecture. A typical home environment is a relatively short-range sensor network.

\subsection{Sensors for Assistive Smart Chair}

As age-related progressive conditions and diseases set in, the elderly might need to change their chair to enable them to get up or sit down as their condition worsens. However, most electric chairs do not offer muscle resistance and are unable to provide personalized services because they simply follow a set cycle to the users resulting in their conditions deteriorating faster. In order to solve this, the sensing techniques [28] has been utilized for the assistive smart chair to capture the user's posture or intention in terms of different significances, subconscious signal extracting, and pattern recognition.

An assistive stand-up robotic chair [28] is developed features 3 DOF motion with an innovative data fusion framework which integrates sensing data acquired from seat, arm, and feet reaction force sensor [29] as in Figure 2. The robotic chair detects assistive person's intention integrating different types of sensors.

The sensing techniques to capture the user's posture or intention can be introduced in terms of different significances, sub-conscious signal extracting, data-fusion, pattern recognition and interpretation, respectively. A haptic sensing chair with applied pattern recognition technology is introduced in capable of classifying the sitting postures into a pre-defined model [30] [31].

In the research of [32], a wheelchair based depressurization system is particularly designed for sedentary patients to eliminate or reduce the pressure concentrated point which could increase the risk of pressure sore. Moreover, in the rehabilitation point of view, motion assistance can be achieved for those patients who intend to change a posture however cannot manage it with their own physical strength. The shifting of subject's centre of gravity (COG) shows the tendency of patient's movement, which can be captured by the pressure distribution sensor through certain algorithm [33]. In the study of manipulating a motorized wheelchair with the patient's own upper body inclination, pressure sensing films are mounted on the seat pan and seat back respectively. The sensing system contributes to recognize the behavioral pattern where the shifting of COG can be extracted, implies the patient's degree of indication. Thus, by moving upper body back and forth the user can drive the wheelchair along longitude direction, while leaning left and right functions as a steering wheel.

\subsection{Sensors Used in Wearable Devices for Fall Detection}

Recent researches estimated that each year, in the U.S., nearly $30 \%$ of elderly people incur in falls, and the 


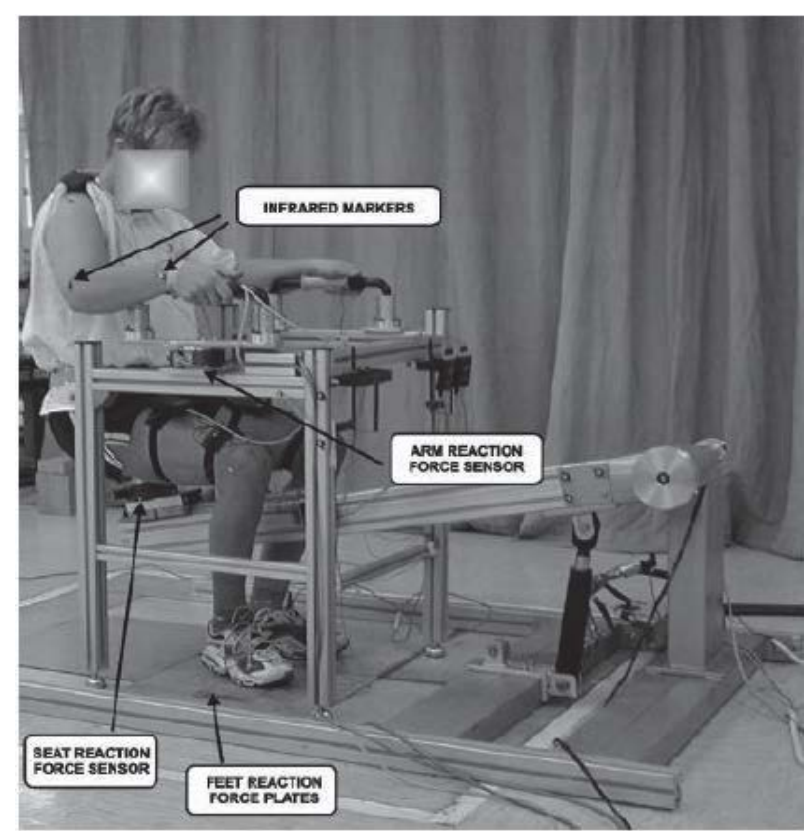

Figure 2. The standing up robotic chair equipped with integrated sensor networks [28].

likelihood of falling increases substantially with age. Falls may directly result in traumas, fractures, permanent disability, or even death. Hence, falling is a major concern for older people due to the higher risk of breakages that results from a lower bone density associated with aging [34]. It is therefore imperative that their support team is alerted in the event of a fall to minimize distress associated with injury. There are devices that seek to monitor an assisted person's status or activities, and devices that provide active support.

iLife fall detection sensor which is worn by the assisted person, may be utilized in conjunction with the Independent LifeStyle Assistant system [10]. This integrates individual devices and augments them with reasoning capabilities enabling the assisted person greater independence. This iLife fall detection sensor not only triggers when abnormal body movements or extended periods of inactivity are detected, but also can be activated manually by pressing a distress button.

In [35], the authors describe two fall sensors, an embedded video-based one and a wearable accelerometerbased one, which can be managed within a data-fusion-oriented framework, implementing policies aimed at maximizing system reliability and minimizing the presence of false alarms. The two sensors can be integrated into a modular architecture to compensate for each other's limits, favoring the development of a harmonic, modular and easily extensible system able to manage different areas of the environment.

The video fall detector is based on a digital camera and a FPGA programmable logic, able to locally process the images and to transmit to a server only aggregated information relating to the 'state of alert', with obvious advantages in terms of end users' privacy. The wearable accelerometer-based sensor is based on a new powerful soft-computing paradigm which makes it possible to extend the task it performs to detecting a whole set of situations, and therefore to make the whole architecture more flexible.

The visual sensor is used to send a central supervision system only aggregated information and not the whole video stream. The sensor output consists only of signals that account for the 'state of alert' on the potential occurrence of a fall. When used in conjunction with other sensors (audio, wearable, etc.), such a compact, economical and little-invasive device can provide a description of the environment being monitored [35]. In [36] the authors speak of their plans to use additional sensors, such as RFID for object localization, floor-mounted vibration sensors for fall detection in privacy-sensitive areas, and infrared cameras, in conjunction with their smart cameras, for additional tracking and health monitoring capabilities.

\subsection{Sensor Network Used in Assistive Technologies}

For speech- and hearing- impaired disabilities, non-verbal form of communication is very important. To alle- 
viate the communication problem among speech-impaired disabilities, an assistive technology that provides a more convenient and less time-consuming means of communication is required. An assistive device for speechand hearing- impaired disabilities has been developed based on the Body Sensor Network (BSN) technology [6]. In the system, real-time recognition of American Sign Language (ASL) fingerspelling gestures is performed based on input signals acquired from a wireless sensor gloves. The recognized gestures will then be mapped into corresponding sounds using speech synthesizer.

In [37], a framework for constructing the fingerspelling gesture recognition model based on the data acquired from a wireless BSN sensor glove is proposed. The glove consists of five flex sensors and a 3D accelerometer providing a measure of finger bending, as well as motion, orientation of the hand, recognition and a speech synthesizer as shown in Figure 3. The flex sensors placed along five fingers are used for detecting finger bending and the 3D accelerometer placed on the back of the hand is used for detecting hand orientation and motion. Data are transmitted to the computer via BSN node placed on the wrist.

In [38], the authors developed a prototype for a working rehabilitation system that would operate safely and accurately in the home of an elderly or recovering patient. The shoe insole and sensor are inserted into the footwear of the user as shown in Figure 4. In this insole, FlexiForce sensor is used to measure the force on the ball and the heel of the foot. The sensors implanted in the legs of the walker are used to collect data to help identify the patient's prognosis during the recovery period. Recovering patients can be monitored while they walk and use their walker in their daily lives. The FlexiForce sensors are used in both the walker and the shoe insole. The sensors aided in the determination of force distribution over the toe of the insole and the legs of the walker. The Tiny Bee Tri-Axis Accelerometer is used in the shoe insole to sense the degree of pronation and supination in the foot. The accelerometer is made by EVBPlus. These sensors output three analog voltages, each proportional the amount of gravitational force experienced along three axes.

In recent years great advancements have been made with prosthetic technology aided by advancements in

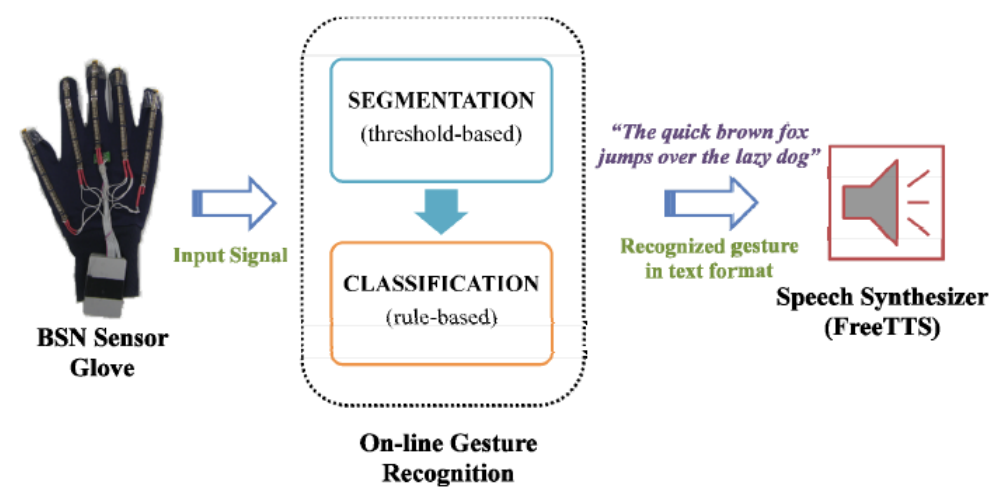

Figure 3. Overall system of the fingerspelling-based speech synthesizing sensor gloves [37].

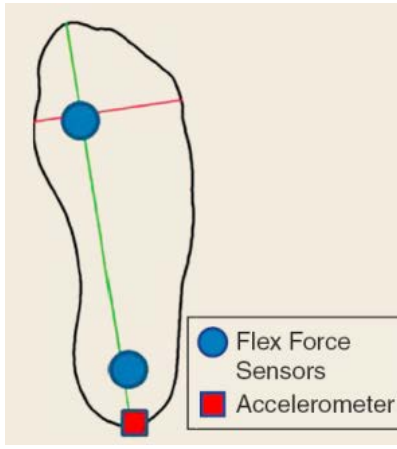

(a)

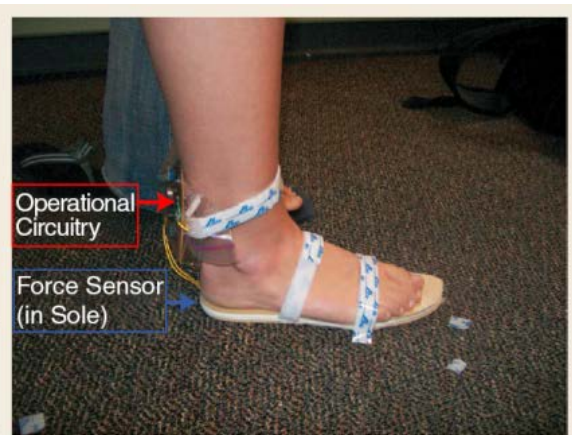

(b)

Figure 4. (a) A schematic of the original insole design; (b) A photograph of the insole [38]. 
materials science and sensors technology. Buckley et al. [39] introduced a sensor suite framework for the partial automation of prosthetic arm control allowing high level control with a reduction of cognitive burden placed upon the user.

A framework is established to use interchangeable sensors to emulate the low level hand-eye co-ordination of a healthy individual. A shoulder mounted depth sensor is used to obtain environment information to be used to locating the target object relative to the prosthesis. Sensors applied in the system include: Laser range finders, stereo cameras, and structured light. When combined together these sensors mimic the natural way in which a user co-ordinates arm movement to a sufficient extent as to allow low level control to be taken over by the system freeing the user into a more high level role.

\section{Conclusion}

This paper has attempted to present a variety of sensors incorporated in human assistive technologies with their features and applications. Through introducing different types of sensors, it is important to point out that sensors used for assistive technologies must consider aspects such as safety, scalability, autonomy, privacy and its impact on the care of the users. Advances in sensors can produce powerful and very rapid movements through a large operational space in development of assistive technologies. Hazard threats arise from unintended contact between assistive devices and humans. Therefore, careful thought needs to be given to hazard assessment while the researchers design the human assistive devices using appropriate sensors. Future research should also consider the interaction between human and assistive devices using sensors to maintain physical and cognitive functional capabilities.

\section{References}

[1] Kothiyal, K. and Tettey, S. (2001) Anthropometry for Design for the Elderly. International Journal of Occupational Safety and Ergonomics: JOSE, 7, 15-34.

[2] Organization for Economic Co-operation and Development (2012) World Population Aging: 1959-2050.

[3] Hoffman, A.H. (2009) Design of Robotic Devices to Assist Persons with Disabilities. IEEE International Conference on Technologies for Practical Robot Applications, Woburn, MA, 1-4. http://dx.doi.org/10.1109/TEPRA.2009.5339653

[4] Marshal, M. (1997) State of the Art in Dementia Care. Centre for Policy on Ageing, UK.

[5] Shi, W.V. and Zhou, M.C. (2011) Recent Advances of Sensors for Pacemakers. IEEE International Conference on Networking, Sensing and Control, 520-525. http://dx.doi.org/10.1109/ICNSC.2011.5874939

[6] Yang, G.-Z., Ed. (2014) Body Sensor Networks. 2nd Edition, Springer-Verlag, London. http://dx.doi.org/10.1007/978-1-4471-6374-9

[7] Tamura, T., Kawarada, A., Nambu, M., Tsukada, A., Sasaki, K. and Yamakoshi, K. (2007) E-Healthcare at an Experimental Welfare Techno House in Japan. Open Medical Informatics Journal, 1, 1-7. http://dx.doi.org/10.2174/1874431100701010001

[8] DoHA (2008) Australian Dementia Resources Guide. Australia.

[9] Vichitvanichphone, S., Talaei-Khoei, A., Kerr, D. and Ghapanchi, A.H. (2014) Adoption of Assistive Technologies for Aged Care: A Realist Review of Recent Studies. 47th Hawaii International Conference on System Sciences, 2706-2715. http://dx.doi.org/10.1109/HICSS.2014.341

[10] Haigh, K.Z. and Kiff, L.M. (2004) The Independent LifeStyle Assistant: AI Lessons Learned. 16th Innovative Applications of Artificial Intelligence Conference, California.

[11] Haigh, K.Z. and Kiff, L.M. (2004) The Independent LifeStyle Assistant (I.L.S.A.): AI Lessons Learned. 16th Innovative Applications of Artificial Intelligence Conference (IAAI), San Jose, California.

[12] Pollack, M.E. (2005) Intelligent Technology for an Aging Population: The Use of AI to Assist Elders with Cognitive Impairment. AI Magazine, 26.

[13] Pollack, M.E. (2005) Intelligent Technology for an Aging Population: The Use of AI to Assist Elders with Cognitive Impairment. AI Magazine, 26.

[14] Hodges, S., Williams, L., Berry, E., Izadi, S., Srinivasan, J., Butler, A., et al. (2006) SenseCam: A Retrospective Memory Aid. UbiComp 2006: Ubiquitous Computing, 177-193.

[15] Hodges, S., Williams, L., Berry, E., Izadi, S., Srinivasan, J., Butler, A., et al. (2006) SenseCam: A Retrospective Memory Aid. UbiComp 2006: Ubiquitous Computing, California, 177-193. 
[16] Cooper, R.A., Boninger, M.L., Cooper, R., Dobson, A.R., Kessler, J., Schmeler, M. and Fitzgerald, S.G. (2003) Use of the Independence 3000 IBOT Transporter at Home and in the Community. Journal of Spinal Cord Medicine, 26, 7985.

[17] Simpson, R.C. (2005) Smart Wheelchairs: A Literature Review. Journal of Rehabilitation Research \& Development, 42, 423-436. http://dx.doi.org/10.1682/JRRD.2004.08.0101

[18] Miller, L.A., Stubblefield, K.A., Lipschutz, R.D., Lock, B.A. and Kuiken, T.A. (2008) Improved Myoelectric Prosthesis Control Using Targeted Reinnervation Surgery: A Case Series. IEEE Transactions on Neural Systems and Rehabilitation Engineering, 16, 46-50. http://dx.doi.org/10.1109/TNSRE.2007.911817

[19] Muzumdar, A. (2004) Powered Upper Limb Prostheses: Control, Implementation and Clinical Application. SpringerVerlag. http://dx.doi.org/10.1007/978-3-642-18812-1

[20] Guizzo, E. and Goldstein, H. (2005) The Rise of the Body Bots. IEEE Spectrum, 42, 42-48. http://dx.doi.org/10.1109/MSPEC.2005.1413730

[21] Chen, N., Tee, K.P. and Chew, C.M. (2013) Assistive Grasping in Teleoperation Using Infra-Red Proximity Sensors. 2013 IEEE RO-MAN, 232-237.

[22] Smith, J., Garcia, E., Wistort, R. and Krishnamoorthy, G. (2007) Electric Field Imaging Pretouch for Robotic Graspers. IEEE/RSJ International Conference on Intelligent Robots and Systems, 676-683. http://dx.doi.org/10.1109/IROS.2007.4399609

[23] Wistort, R. and Smith, J. (2008) Electric Field Servoing for Robotic Manipulation. IEEE/RSJ International Conference on Intelligent Robots and Systems, 494-499. http://dx.doi.org/10.1109/IROS.2008.4650721

[24] Hsiao, K., Nangeroni, P., Huber, M., Saxena, A. and Ng, A.Y. (2009) Reactive Grasping Using Optical Proximity Sensors. IEEE International Conference on Robotics and Automation, 2098-2105. http://dx.doi.org/10.1109/ROBOT.2009.5152849

[25] Kim, Y., Park, K., Bang, W., Kim, M., Han, J. and Bien, Z.Z. (2002) Development of Intelligent Bed-Robot System for the Elderly or the Disabled. 3rd International Workshop on Human-friendly Welfare Robotic Systems, Daejeon, Korea, 94-98.

[26] Teichmann, M. and Mishra, B. (2000) Reactive Robotics i: Reactie Grasping with a Modified Gripper and Multifingered Hands. I. J. Robotic Res., 697-708.

[27] R. Yu, G.Z. Yang, B.P.L. Lo, “Autonomic body sensor networks,” 2014 IEEE MTT-S International Microwave Workshop Series on RF and Wireless Technologies for Biomedical and Healthcare Applications, pp. 1-3, 2014. http://dx.doi.org/10.1109/IMWS-BIO.2014.7032412

[28] Lu, H., Li, D., Oyekan, J. and Maple, C. (2013) A Survey on Assistive Chair and Related Integrated Sensing Techniques. International Conference on Manipulation, Manufacturing and Measurement on the Nanoscale, Suzhou, China, 129-134. http://dx.doi.org/10.1109/3M-NANO.2013.6737398

[29] Kamnik, R. and Bajd, T. (2004) Standing-Up Robot: An Assistive Rehabilitative Device for Training and Assessment. Journal of Medical Engineering and Technology, 28, 74-80. http://dx.doi.org/10.1080/0309190032000112306

[30] Tan, H.Z., Slivovsky, L.A., Member, S. and Pentland, A. (2001) A Sensing Chair Using Pressure Distribution Sensors. IEEE/ASME Transactions on Mechatronics, 6, 261-268. http://dx.doi.org/10.1109/3516.951364

[31] Silvovsky, L.A. and Tan, H.Z. (2000) A Real-Time Static Posture Classification System. Proceeding of the ASME Dynamic Systems and Control Division, 2, 1049-1056.

[32] Chugo, D., Fujita, K., Sakaida, Y., Yokota, S. and Takase, K. (2011) Development of Depressurization System for a Seated Patient on a Wheelchair. 2011 IEEE International Conference on Mechatronics, 615-620. http://dx.doi.org/10.1109/ICMECH.2011.5971189

[33] Lu, H., Li, D., Oyekan, J. and Maple, C. (2013) A Survey on Assistive Chair and Related Integrated Sensing Techniques. International Conference on Manipulation, Manufacturing and Measurement on the Nanoscale, 129-134. http://dx.doi.org/10.1109/3M-NANO.2013.6737398

[34] O’Brien, A. and Mac Ruairi, R. (2009) Survey of Assistive Technology Devices and Applications for Aging in Place. Second International Conference on Advances in Human-oriented and Personalized Mechanisms, Technologies, and Services, Porto, 7-12. http://dx.doi.org/10.1109/CENTRIC.2009.9

[35] Cagnoni, S., Matrella, G., Mordonini, M., Sassi, F. and Ascari, L. (2009) Sensors Fusion-Oriented Fall Detection for Assistive Technologies Applications. 9th International Conference on Intelligent Systems Design and Applications, 673-678. http://dx.doi.org/10.1109/ISDA.2009.203

[36] Williams, A., Xie, D., Ou, S., Grupen, R., Hanson, A. and Riseman, E. Distributed Smart Cameras for Aging in Place.

[37] Vutinuntakasame, S., Jaijongrak, V.R. and Thiemjarus, S. (2011) An Assistive Body Sensor Network Glove for Speech- and Hearing-Impaired Disabilities. International Conference on Body Sensor Networks (BSN), 7-12. 
http://dx.doi.org/10.1109/BSN.2011.13

[38] Mellodge, P. and Vendetti, C. (2011) Remotely Monitoring a Patient's Mobility: A Digital Health Application. IEEE Potentials, 33-38. http://dx.doi.org/10.1109/MPOT.2010.939453

[39] Buckley, M., Vaidyanathan, R. and Mayol-Cuevas, W. (2011) Sensors Suites for Assistive Arm Prosthetics. 24th International Symposium on Computer-Based Medical Systems, 1-6. http://dx.doi.org/10.1109/CBMS.2011.5999153 